\title{
Một số kết quả trên phụ thuộc mạnh
}

\author{
Vũ Dức Thi \\ Viẹn Coòng nghệ thông tin
}

\section{Mở đẩu}

Phụ thuộc mạnh được dề xuất và tiên đî́ hóa trong $[2,3,1]$ và đà được cài đặt trong hệ thiết kế các hệ quàn trị cơ sơ dữ liệu có đặc tính nồi bật thuận lợi là phân tácle các màng dừ liệu lớn thành các màng dự liệu uhỏ hơn. Bài bảo này trinh bày các kết quả mới thu được vể phụ thuộc mạnh. Trước tiên, chúng tôi dưa ra thuật toản tím bao đóng cua một thuộc tinh với dộ phức tạp là tuyến tính $O(p)$, ở đây $p=l(S)$ - chiều dài cua tập phụ thuộc mạnh $S$. Dựa vào thuật toán này, chúng tôi đưa ra thuật toán giải quyết bài toán thành viên. Khải niệm khóa dược dưa ra với ý nghỉa xác định duỵ nhất một dối tương. Chúng tôi đưa ra thuật toán tím một khóa với độ phức tạp là đa thực, chúng tôi cûng chi ra dược răng đối với phụ thuộc mạnh mọi klıóa đồu có lực lượng như nhau và bài toán tỉm tập tất cà các khóa tối thiểu cria $S(K s)$ dược giài quyết bằng một thuật toán có độ phức tạp là đa thức nếu lực lượng cưa $r i s$ là đa thức.

\section{Các định nghĩa}

Dịnh nghia 1.1: Cho $R=\left\{h_{1}, \ldots, h_{n}\right\}$ là quan lệ trên $\Omega$ và $A, B \subseteq \Omega$. Chúng ta nơi rằng $B$ phụ thuộc mạnh vào $A$ trong $R\left(\right.$ kí hiệu $\left.A \frac{S}{R} B\right)$ nếu

$$
\left(\forall h_{i}, h_{j} \in R\right)\left((\exists a \in A)\left(h_{i}(a)=h_{j}(a)\right) \rightarrow(\forall b \in B)\left(h_{i}(b)=h_{j}(b)\right)\right) .
$$

Đặt $S_{R}=\left\{A \frac{S}{R} B\right\} . S_{R}$ được gọi là họ đủ các phụ thuộc mạnh của $R$. 
Dịnh nghia 1. Q: Cho $R$ là quan hệ trên $\Omega . A, B \subseteq \Omega$. Chúng ta nói $A \rightarrow B$ là một phụ thuộc mạnh của $R$ nếu $B$ phụ thuộc mạnh vào $A$ trong $R$, hay $A \underset{R}{\rightarrow} B$. Chúng ta cũng nọi rằng $R$ thỏa mãn $A \rightarrow B$.

Dịnh nghĩa 1.3: Cho $S$ là một tập phụ thuộc mạnh trên $\Omega$ và $X \rightarrow Y$ là một phụ thuộc mạnh, với $X, Y \subseteq \Omega$. Chúng ta nói $S$ kéo theo logic ra $X \rightarrow Y$, và viết $S \vDash X \rightarrow Y$ nếu với mọi quan hệ $R$ trên $\Omega$ thóa mãn các phụ thuộc mạnh trong $S$ thì cũng thỏa mãn phụ thuộc mạnh $X-Y$.

Dịnh nghĩa 1.4: Cho $\Omega$ là một tập hữu hạn các thuộc tính, với $\forall A, B, C, D \subseteq \Omega, a \in \Omega$. Các $S$ - luật suy dẫn là:

$$
\begin{aligned}
& S 1:\{a\}-\{a\} ; \\
& S 2: \text { Nếu } A \rightarrow B \text { và } B \rightarrow C \text { với } B \neq \emptyset \text { thì } A \rightarrow C ; \\
& S 3: \text { Nếu } A \rightarrow B \text { và } C \subset A, D \subseteq B \text { thì } C-\dot{D} ; \\
& S 4: \text { Nếu } A \rightarrow B \text { và } C-D \text { thí } A \cup C \rightarrow B \cap D ; \\
& \dot{S 5}: \text { Nếu } A \rightarrow B \text { và } C \rightarrow D \text { với thì } A \cap C \rightarrow B \cup D ;
\end{aligned}
$$

Dê dàng nhận thấy rằng $S_{R}$ thỏa mã các $S$-luật suy diễn, do vậy các $S$-luật suy diển này cho phép ta suy diên ra tất cảcác phụ thuộc nạ̣nh khác tự $S$.

\section{Bao đóng của tập phụ thuộc mạnh, bao dóng của các thuộc tính}

Dịnh nghia 2.1 : Cho $S$ là tập các phụ thuộc mạnh trên $\Omega$. Đặt $S^{+}$là bao đóng của $S$, là tập tất cả các phụ thuộc mạnh được kéo theo logic từ $S$, có nghĩa là

$$
S^{+}=\{X-Y: S \vDash X \rightarrow Y\} \text {. }
$$

Chưng ta không hy vọng liệt kê toàn bộ $S^{+}$, nhưng ít ra. chụ́ng ta có thể biết được khi nào một phụ thuộc mạnh $X \rightarrow Y$ cho trước thuộc vào $S^{+}$, hay được suy diên ra tir $S$.

Trước hết, chúng ta đưa ra khái niệm bao đóng của tập các thuộc tỉnh ứng với tập các phụ thuộc mạnh đà cho.

Dịnh nghĩ $2.2:$ Cho $S$ là tập các phụ thuộc mạnh trên $\Omega, X$ là tập con của $\Omega, A \subseteq \Omega$. Chúng ta gọi $X^{+}$, bao đóng của $X$ ứng với $S$, là tập các thuộc tính $a$ sao cho $X \rightarrow\{a\}$ có thề suy diễn tîr $S$ bới các $S$-luật suy diễn, có nghĩa là: $X^{+}=\left\{a \in \Omega: X^{*} \rightarrow\{a\} \in S^{+}\right\}$.

Ta có bồ đề sau:

Bố đề 2.3: Phu thuộc mạnh $X \rightarrow Y$ được suy diễn tì̛ $S$ bởi các S-luật suy diễn nếu và chì nếu $Y \subseteq \mathrm{X}^{+}$.

Chứng minh. Gỉa sứ $Y=a_{1} a_{2} \ldots a_{k}$ với $a_{i} \in \Omega, i=\overline{1, k}$. Nếu $Y \subseteq \Omega$, do định nghĩa của $X^{+}$, ta có $X \rightarrow\left\{a_{i}\right\}$ với $i=\overline{1, k}$. Vì vậy theo $S 5$ ta có $X \rightarrow Y$. Ngược lại, gía sư $X \rightarrow Y$ được suy diễn từ $S$ bời các $S$-luật suy diễn. Với mồi $i=\overline{1, k}, X \rightarrow\left\{a_{i}\right\}$ là đúng bởi (.S3). Vậy $Y \subseteq x^{+}$. Bồ đề đi̛ợc chứng minh. 
Liĉn quan đến khái niệm bao dóng và các tỉnh chất cria bao*đóng, chúng ta dưa ra các hàm nậnh.

Dịnh nghia 9.4 : ('ho $\Omega$ là một tập hừu hạn, ánh xạ $F: P(\Omega)-P(\Omega)$ đurợc gọi là hàm mạnh trền $\Omega$ nếu với mọi $a b \in \in$ b và $A \subseteq \Omega$, các tính chất sau là đúng:

(1) $a \in F(\{a\})$.

(2) $b \in F(\{a\})-F(\{b\}) \subseteq F(\{a\})$.

(3) $F(A)=\bigcap_{a \in A} F(\{a\})$.

Nhận xét 2.5: Dể dàng nhận thấy răng các tỉnh chất sơ đẳing sau cuia hàm mạnh là dúng

(1) Với $A, B \in P(\Omega)$, ta có $F(A \cup B)=F(A) \cap F(B)$;

(2) Bới quy ước $\cap \emptyset=\Omega$. 1a có $F(\theta)=n \emptyset=\Omega$;

(3) Với $A \subseteq B$, thì $F(B) \subseteq F(A)$.

Bồ đề 2.6: Cho S là tập rác phụ thuộc mạnh trến $\Omega$ và $A \subseteq \Omega$. Chüng ta xây dụng ng inh $x a F_{\mathrm{s}}: P(\Omega) \rightarrow P(\Omega)$ uhu sau:

$$
F_{s}(A)=\left\{a \in \Omega: \quad 1 \rightarrow\{a\} \in S^{+}\right\}
$$

thi Fs là một hì mạnh.

Chứng minh. Gia sư $S$ là tập các phụ thuộc mạnh. ta có $F_{s}(\{a\})=\{b \in \Omega:\{a\}-$ $\left.\{b\} \in S^{+}\right\}$do luật $(S 1)$, ta có $a \in F_{s}(\{a\})$. Do dịnh nghía của $F_{s}$ và luật $(S 5)$, ta có $\{b\}-F_{s}(\{b\})$ và nếu $b \in F(\{a\})$ thí $\{a\}-\{b\}$. Do vậy, ta có $\{a\}-\{b\}$ và $\{b\} \rightarrow F_{s}(\{b\})$, sư dụng luật $(S 2)$, ta dược $\{a\}-F_{s}(\{b\})$ và $F_{s}(\{b\}) \subseteq F_{s}(\{a\})$ do dịnh nghia của $F_{s}$.

Cho $A$ là tập các thuộc tiul, ta có $A-F_{s}(A) \in S^{+}$, do vậy với mọi $a \in A$, ta có $\{a\} \rightarrow F_{s}(a)$ bời luật $(S 3)$ do dó $F_{s}(A) \subseteq F_{s}(\{a\})$ do dịnh nghía cùa $F_{s}(\{a\})$. Vì vậy,

$$
F_{s}(1)=\bigcap_{a \in A} F_{x}(\{a\})
$$

Vậy $F$ s là một hàm mạnh.

Nhận xét 2.7. Dể dàng nhận thấy răng $F_{s}(A)$ chính là bao đóng của tập A các thuộc tinh ứng với tập các phụ thuộc mạnh $S$ trên $\Omega$ do dịnh uighia cúa $F$ và dịnh nghía bao dóng cua một tập thuộc tinh, có nghia lit $A^{+}=F_{s}(A)$ hay $A^{+}=\bigcap_{a \in A}\{a\}^{+}$. Do vậy dể tỉnh bao đóng cua một tập thuộc tỉnh, trước hết ta cân phài tính bao đóng cua mọi thuộc tỉnh nằm trong thuṇc tính dó.

Thuật toán 2.8: Iìm bao dóng cria một thuộc tính cho trước ứng với tập các phụ thuộc mạnh đà cho:

Vào: Tập hừu hạn các thuộc tính $\Omega$, tập hiru hạn các phụ thuộc mạnh $S$ và $a \in \Omega$. Ra: $\{a\}^{+}$, bao đóng cua $\{a\}$ ứng với $S$. 
Cách tỉnh: Chúng ta tỉnh dãy các tập thuộc tỉnh $X^{(0)}, X^{(1)}, \ldots$ theo quy tắc saul:

1. $X^{(0)}=\{a\}$,

2. $X^{(i+1)}=X^{(i)}$, nếu có một ph thuộc mạnh $Y \rightarrow Z \in S$ nào đó sao cho $Y \cap X^{(i)} \neq \emptyset$ và $A=Z \backslash X^{(i)} \neq \emptyset$, và $X^{(i+1)}=X^{(i)}$, trong trường hợp ngược lại.

Do vậy, chúng ta sẽ có một dãy các tập bao nhau $X^{(0)} \subseteq X^{(1)} \subseteq \ldots \subseteq X^{(i)} \subseteq X^{(i+1)} \ldots \subseteq$ $\Omega$ và vì $\Omega$ là hưuu hạn, các tập phụ thuộc mạnh là hữu hạn, nên chưng ta sẽ đi đến $i$ mà $X^{(i)}=X^{(i+1)}$. Điêuu đó kéo theo rằng $X^{(i)}=X^{(i+1)}=X^{(i+2)} \ldots$

Khi đó chúng ta lấy $\{a\}^{+}=X^{(i)}$.

Nhận xét 2.9: Thuật toán có thề cài đặt đề chạy trong thời gian tỷ lệ số các phụ thuộc mạnh trong $S$ và chiều dài các phụ thuộc mạnh dó (số các thuộc tỉnh trong các phụ thuộc mṣnh của $S$ ). Do vậy, thuật toán tỉm bao đóng của một thuộc tînh cho trước có độ phức tạp là tuyến tỉnh $O(p)$, ở đây $p=l(S)$ ( $p$ là chiếu dài của $S$, tức là số các thuộc tỉnh xuất hiện trong $S$ kề cà số lần lặp các thuộc tính giống nhau).

Chúng ta phài chỉ ra rằng thuật toán trên là đúng. Dê dàng nhận thấy rằng với mọi $i: X^{(i)} \subseteq\{a\}^{+}$nhưng ngược lại thì khó hơn.

Dịnh lý 2.10: Thuật toán 2.8 tính đuing $\{a\}^{+}$.

Chứng minh. Trước tiên chúng ta chì ra bằng quy nạp theo $j$ rằng $X^{(j)} \subseteq\{a\}^{+}$. Гa phài chứng minh mệnh đề trên thỏa màn 2 điêu kiện:

1. Với $j=0$ thì $X^{(0)} \subseteq\{a\}^{+}$.

Thực vậy, $X^{(0)}=\{a\}$, do đó hiển nhiên là $X^{(0)} \subseteq\{a\}^{+}$.

2. Cho $j \geq 1$, già sừ rằng $x^{(j-1)} \subseteq\{a\}^{+}$, ta phài chưong minh rằng $X^{-(j)} \subseteq\{a\}^{+}$.

Ta chi ra cho trường hợp ngược lại, nếu $b \in\{a\}$ thi $b \in X^{(j)}$ nào dó. Thuật toán (2.8) kết thúc trước khi tînh $X^{(j)}$ hay không là không quan trọng bời vi nếu thuật toán này dừng khi $X^{(i)}=X^{(i+1)}$ với $i<j$ thî chúng ta có $X^{(i)}=X^{(j)}$. Trong thực tế ta có $i \leq m$, với $m$ là phụ thuộc mạnh của $S . m=|s|$, vì chì có $\dot{m}$ phụ thuộc mạnh trong $S$ thôi.

Chúng ta sè chì ra rằng nếu $\{a\}-Y \in S, 1$ ức là $\{a\}-Y$ được suy dièn từ $S$ bời các $S$-luật suy diễn, thí nếu $b \in Y$, ta phài có $b \in X^{(j)}$ nào đó. Chứng minh được tiến hành bằng phương pháp quy nạp theo số dòng suy diên ra $\dot{Y}$ tì $S$. Ớ đây mời dòng là một phụ thuộc mạnh cua $S$, hoặc là một phụ thuộc mạnh được suy diễn từ các dòng trước và dòng cuối cùng là $\{a\}-Y$.

Chúng ta chứng minh hai điều kiện sau:

1. Với 1 dòng:

- $\{a\} \rightarrow Y \in S$, vì $\{a\} \cap X^{(j-1)}=\{a\} \neq \emptyset$ nên theo thuật toán $(2.8)$ sè phài tổn tại một $j>0$ nào đó sao cho hoặc là $A=Y \backslash X^{(j-1)} \neq \emptyset$ thì $X^{(j)}=X^{(j-1)} \cup A$ hoặc là $Y \subseteq X^{(j)}$ do đó $Y \subseteq X^{(j)}$.

- $\{a\}-Y$ dược suy diền bới $S 1$, tức là $Y=\{a\}$ thì hiễn nhiên là $Y \subseteq X^{(0)}$. 


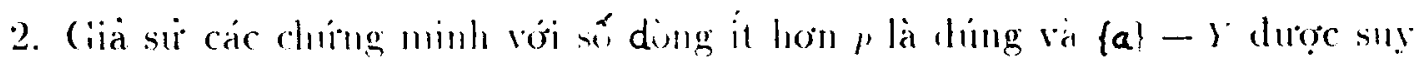
dion tir st bơi p dong. kionin 1.

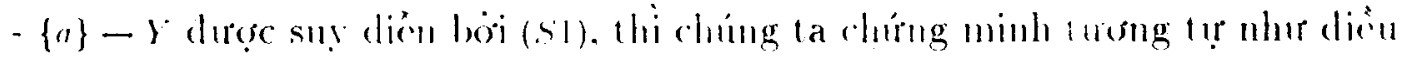

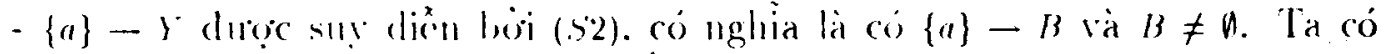

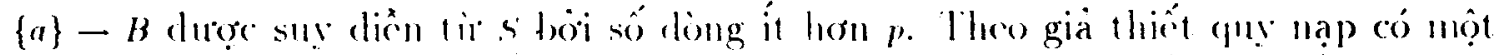
$x^{(j)}$ nào dó $\left.d \hat{\hat{c}} l\right] \subseteq x^{(j)}$.

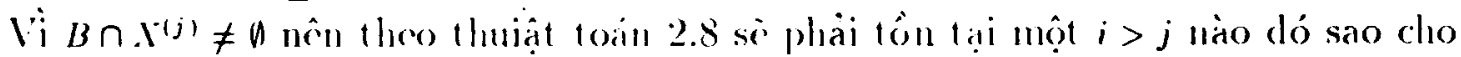
hoặc là $.1=Y \backslash x^{(i-1)}=n$ thi $x^{(i)}=x^{(1-1)} \cup A$ hoặc là $\gamma \subseteq x^{(i)}$, do dó $Y \subseteq x^{(i)}$.

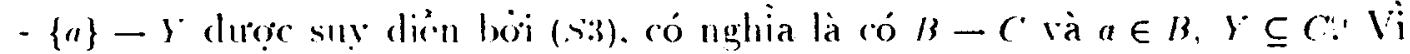
$B \cap \gamma^{-(0)}=\{a\} \neq n$ nên lheo lhuạt toin 2.8 so phäi tôn tại một $j>0$ nào dó sao cho

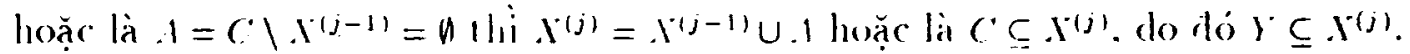

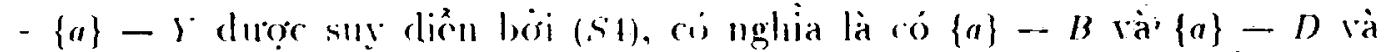

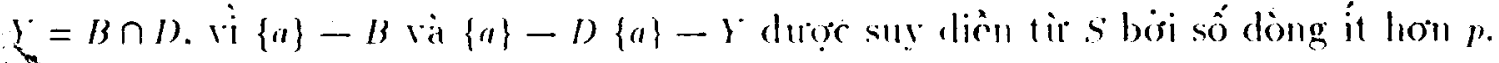
Do vậy, ta có $\xi^{\circ} \subseteq x^{-(j)}$.

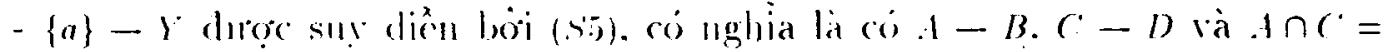

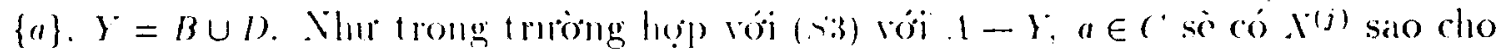

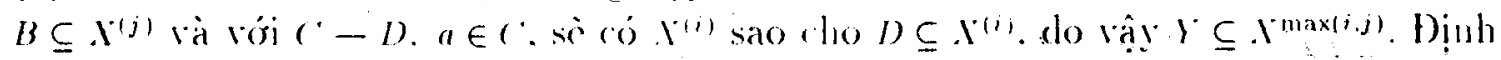
lý duợc clựng minh.

Thuật toán 2.11: Bài tocin thành viôn

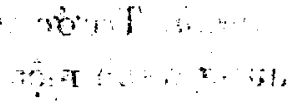

Vào: lập hừu hạn các thuộc tinlı !?. tập hữu hạn các phụ thuộc mạnh $S$ và phụ lhuọc mạnh $x-y$.

Ra: $x-y^{*} \in S^{+}$?

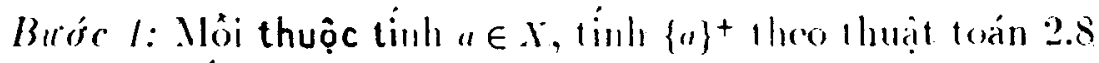

$(O(n p))$

Buróc s: 'Tinh $x^{+}=\cap\{a\}^{+} \quad\left(O\left(n^{-}\right)\right)$

Bức 3. Kiền tra $r \subseteq x^{+?}$ ? \& $(O(n))$

Chúng minh. Theo bồ dé 2.3 ta ci $x-\gamma^{*} \in \mathrm{s}^{+} \Leftrightarrow \gamma^{\prime} \subseteq \mathrm{x}^{+}$.

Nhận xét 2.12: Bài toán lhành viên vởi tập S rảc phụ thuộc mạnh trên $\Omega$ và phụ

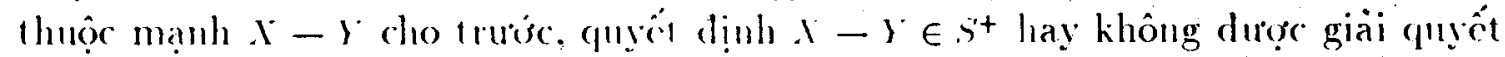

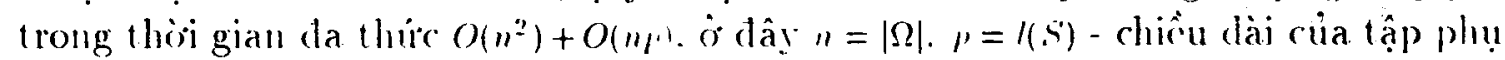
thuộc mạnh $s$.

\section{Khóa}

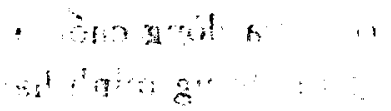

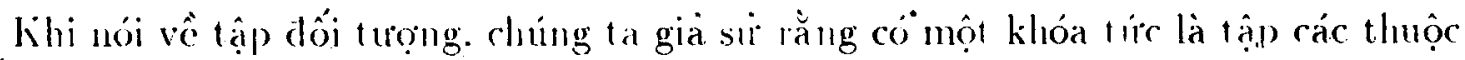
tính mà nó xác dịnh dụ uhất một rối tượng.

Dịnh nghia 3.1: Cho $R$ là quan hệ trên $\Omega$, và $K \subseteq \Omega$. ('huing ta nói $K$ là khoá tối tiều ciia $R$ (Khóa tối tiều cia $S$ ) nốu: 
Đặt $K=\left\{a_{1}, a_{2}, \ldots, a_{k}\right\} . F_{H}\left(\left\{a_{j}\right\}\right)=\left\{a \in \Omega:\left\{a_{j}\right\} \frac{R}{S}\{a\}\right\} . F_{s}\left(\left\{a_{j}\right\}\right)-\left\{a \in \Omega \cdot\left\{a_{j}\right\}-\right.$ $\left.\{a\} \in S^{+}\right\}$.

1.

$$
\bigcup_{j=1}^{k} F_{k}\left(\left\{a_{j}\right\}\right)=\Omega\left(\bigcup_{j=1}^{k} F_{*}\left(\left\{a_{j, j}\right\}\right)=\Omega\right)
$$

2. Với mọi tập con thục sụ $K^{\prime}$ cria $K$ thi $\underset{a, \in K}{\cup} F_{h}\left(\left\{a_{j}\right\}\right) \subset \Omega\left(\underset{a, \in K}{\cup} F_{s}\left(\left\{a_{j}\right\}\right) \subset \Omega\right)$. Ta nhận thấy rới mối quan hệ hay tập các phụ thuộc mạul, có thể là một hay nlicêu khóa tối tiều. Lí hiệu $K_{R}\left(K_{s}\right)$ là lập tất cà các khóa tối tiếu cua $R(S)$. Rô ràng rằng $K_{R}, K$, là các hệ Sperner trên $\Omega$. if

Thuật toán 3.2: Tỉm một klóa tối tiều cua tập các phụ thuộc mạnh $S$ trên $\Omega$.

Vào: Tập hưu hạn các thuộc timh $\Omega$, tập hừu hận các phụ thuộc mạnh $S$ trền $\Omega$.

Ra: $K \in K_{:}$.

Cách tịm: Già sü $\Omega=\left\{a_{1}, a_{2} \ldots a_{n}\right\}$.

Vợi mọi $a_{i}, i=\overline{1, n}$, si dụng thuạt toán 2.8 tinh $F_{s}\left(\left\{a_{i}\right\}\right)=\left\{a_{i}\right\}^{+}$

1)ặt $k_{0}=\Omega$

$$
\kappa_{i}=\left\{\begin{array}{lll}
\kappa_{i-1} \backslash\left\{a_{i}\right\} & \text { nốu } \exists a_{j} \in K_{i-1} \backslash\left\{a_{i}\right\} \mid a_{i} \in F_{*}\left(\left\{a_{j}\right\}\right) & \left(O\left(n^{3}\right)\right\} \\
\kappa_{i-1} & \text { nếu ngự̛̣ lại }
\end{array}\right.
$$

thì $K_{n}$ chinhl là khóa tối tiếl cua $S$ tức là $K_{n} \in K_{s}$.

Cháng minh. Do rách xậ̀ dụng $K_{j}$. ta có

$$
\bigcup_{a_{i} \in K,} r\left(\left\{a_{i}\right\}\right)=!, \forall j=\overline{1, n}
$$

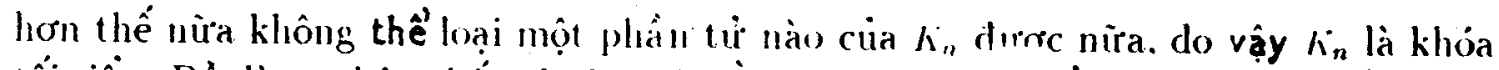
tổi tiều. Dể dàng nhận thậ̣ thuật luán tim một khóa tôi tiểu dược giăi quyết trong

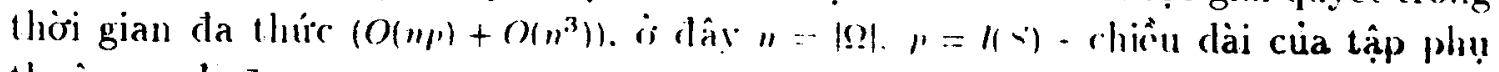
throor manhes.

1)ịnh nghia 3.3: (ho $S$ là tập các phụ thuộc mạnh trên $\Omega$ và $a, b$ là các thuộc tỉmh cua $\Omega$. Chuing ta nói rằng a và b là tương dương (kí hiệu $\equiv)$ nếu $F_{s}(\{a\})=F_{s}(\{b\})$.

Ki hiệu $L(a)=\{b \in \Omega: \dot{b} \equiv a\}$."

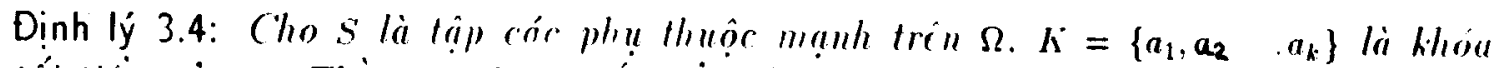

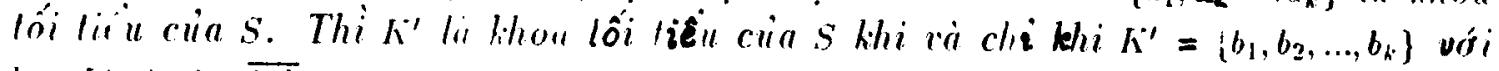
$b_{i} \in L\left(a_{i}\right), i=\overline{1, k}$.

Chi̛ng minh. Do dịnh nghỉa cua khóa tổi tiểı và định nghỉa phẩn từ tương dirơng, dể dàng nhận thấy $K^{\prime \prime}$ là khóa tối tị̂u clia $s$. Ngược lại già sừ $K^{\prime \prime}=\left\{c_{1}, \ldots, c_{1}\right\}$ là 
khóa tối lie u cia s Dặt $l=\Omega \backslash \bigcup_{i=1}^{n} L\left(a_{1}\right)$. Xà $a_{j} \in L$, vì $a_{j} \notin K$ nên phäi tồn tại $a_{i} \neq a_{j}$ sao cho $a_{j} \subseteq F_{s}\left(\left\{a_{i}\right\}\right)$ và $F_{s}\left(\left\{a_{j}\right\}\right) \neq F_{s}\left(\left\{a_{i}\right\}\right)$. Vì $k^{*}$ là khóa tối tiêu của $S$, có nghia là $\bigcup_{i=1} F_{s}\left(\left\{a_{i}\right\}\right)=\Omega$. do vậy tồn $t$ ại $c_{p}$ dề $a_{i} \in F_{s}\left(\left\{c_{p}\right\}\right)$. Vì vậy. ta có $F_{s}\left(\left\{a_{i}\right\}\right) \subseteq F_{s}\left(\left\{c_{n}\right\}\right)$ và $a_{j} \in F_{s}\left(\left\{c_{p}\right\}\right), a_{j} \neq c_{p}$ và $F_{s}\left(\left\{a_{i}\right\}\right) \neq F_{s}\left(\left\{c_{p}\right\}\right)$. Do vậy $a_{j} \notin K^{\prime \prime}$, và $K^{\prime \prime}$ không chứa $L$. Do dó sè tồn tại $b_{1}, \ldots b_{k}$ với $b_{i} \in L\left(a_{i}\right), i=\overline{1, k} đ \hat{\hat{c}} K^{\prime \prime \prime}=\left\{b_{1}, \ldots, b_{k}\right\}$ với $b_{i} \in L\left(a_{i}\right), i=\overline{1 . k}$. Dịnh lý dược chứng minhl.

Nhận xét 3.7: Các bướ $1,2,3$ cua thuật toán trên chỉ đòi hỏi thới gian là đa thức dối với $\Omega$ và chijiểu dài của các tập phụ thuộc mạnl $S$. ̛̛̉ clây $n=|\Omega|$ và $p=\|(S)$. Do vậy bài toán tîn tập tất cà cảc khóa tối tiểu của $S$ được giải quyết bắng một thuật toán có độ phực tạp là đa thức nếu lược lượng củK, là đa thức.

\section{Tài liệu tham khảo}

1. Armstrong W.W., Dependency structures of data base relationships, Information Processing 74, Holland Publ. Co. 197.1, p. 580-58:3.

2. Czédli G.. On dependenriss in thr relational model of data, EIK v. 17, 1981, p. 103-112.

3. Demetrovics J., Relácios adatmodrll logikai es structurális vizsgálata MTA-

SZTAKI Tanulmányok, Budapest, t. 114, 1980, p. 1-97.

4. Demetrovics J. \& Gycpesi G., On the funclional de pendency and some generalizations of it, Acta Cybernetica Hungary V. :3, 1983, p. 295-305.

5. Demetrovics J \& Vu Duc Thi, Relations and minimal keys, Acta Cybernetica v. 8. 1988 , p. $279-285$.

6. Vu Duc Thi, Strong deprndencies and s-semilallicrs, Acta Cybernetica v. 8, 1987. p. $175-202$.

\section{Abstract \\ Some results on strong dependencies}

Strong dependeneirs hare been introduced and ariomatized in [2.3.4]. In this paper, we give some results, that are related to slrong df pendencies. Wi give an algorithm for computing closures of one attribute in lincast arme $O(p)$ where $p$ is the length of $S$, and then we give a me mbership algorithm. We also give an alyorithm for finding a minimal key and finding a set of all minimal keys of $S$. 